\title{
Resource allocation for spectrum-leasing based CRN with delay-sensitive traffic
}

\author{
Yanbo Ma*, Xiao Yin, Xiao Yang and Qiang Liu
}

\begin{abstract}
In this paper, cooperative resource allocation strategies are characterized for a spectrum-leasing based cognitive radio network (CRN), where the primary system leases the licensed band to the secondary system for a fraction of time in exchange for the secondary user (SU) acting as relay. Here, both amplify-and-forward (AF) and decode-and-forward (DF) relay protocols are considered. Considering the delay-sensitive traffic in CRN, the proposed strategies ensure delay provisioning for both primary user (PU) and SU with multiple system design objectives. In particular, we propose a multi-objective optimization framework, which incorporates two important system design objectives: the average sum power minimization and the leased time minimization. By integrating information theory with the concept of effective capacity, the adopted multi-objective optimization problem is recast as a convex optimization one via employing weighting method and sequentially solved by applying the Lagrangian dual method. It is shown that the global optimal solution of the original problem is characterized by a Pareto set which provides a quantitative insight into the tradeoff between the transmit power and leased time. Moreover, to learn the statistics of the wireless channels on the fly, we also put forward a stochastic iterative algorithm to achieve the optimal power and time allocation by employing the stochastic optimization theory. Numerical results not only reveal the nontrivial tradeoff among the considered conflicting system design objectives but also demonstrate that the proposed strategies perform better in saving wireless resources than existing resource allocation policies for different Quality-of-Service (QoS) exponent sets, especially when the delay requirement is strict.
\end{abstract}

Keywords: Spectrum-leasing based CRN, Resource allocation, Effective capacity, Multi-objective optimization, Stochastic optimization, Delay provisioning

\section{Introduction}

Dynamic spectrum sharing (DSS) in cognitive radio networks has been widely considered as an effective means to allow the secondary users to dynamically access the licensed frequency bands and thereby to overcome the problem of spectrum under-utilization caused by the static spectrum allocation. In $[1,2]$, the dynamic spectrum leasing was proposed as a new paradigm for DSS mechanisms, in which the secondary system is granted to use the licensed frequency bands by the PU in exchange for cooperation. The incentive for PUs to lease their licensed bandwidth is that they will benefit from the enhanced QoS thanks to cooperation with SUs. The spectrum-leasing

\footnotetext{
*Correspondence: yanboma@sdufe.edu.cn

Department of Network and Information Security, Shandong University of Finance and Economics, Jinan, China
}

based cooperation can guarantee the benefits of both PUs and SUs simultaneously, achieving a "win-win" situation.

On the observations of the selfish properties of users and the fact that the time for SU to access the licensed band is obtained as a revenue for relaying the primary traffic, there exists competition among PUs and SUs for finite wireless network resources. And with the help of the information theory, there has been extensive research on how to guarantee the benefits of spectrum-leasing based CRN via optimally allocate the wireless resources among PUs and SUs [3-18], and a good summary of the state of the art is provided in $[3,4]$. Thereinto, the cooperation among PUs and SUs was commonly modeled by the convex optimization problem $[5,6]$ where the globally optimal resource allocation is analytically derived, or by employing the widely used economical concepts, such as a Stackelberg game [2], a Nash Bargaining game [7-9], contract theory $[10,11]$, auction theory $[12,13]$, matching theory 
[14], etc. For example, aiming to optimally calculate the design parameters of the primary and secondary networks, [5] has considered the problem of maximizing the smaller of secondary transceiver average rates, while preserving a minimum rate for the primary pair. Lu et al. [6] studies the joint optimization of the set of subcarriers used for cooperation, subcarrier pairing, and power allocation such that the transmission rate of the secondary system is maximized, while helping the primary system to achieve its target rate. Toroujeni et al. [12] proposes an auction framework in which the PU tries to find the optimum amount of resources (in both time and frequency) leased to SUs, whereas each SU's goal is to find the optimum power level to maximize its own profit. Inspired by the matching approach, in [14], the network is modeled as a matching market, where each PU puts forward a proposal representing a combination of relay power and spectrum access time to attract SUs, while each SU maximizes its utility by selecting the most suitable PU.

Nevertheless, all the aforementioned models cannot be directly applied to the spectrum-leasing based CRN scenario with the delay-sensitive traffic, since Shannon theory places no restriction on the delay of the transmission scheme achieving capacity. Although some works $[17,18]$ have been done for the delay-sensitive traffic, the delay metric is considered in the context of the deterministic delay guarantee, which is practically infeasible for wireless networks due to the time-varying nature of wireless channels. To address this issue, the statistical delay provisioning is adopted to ensure a small steady-state delay violation probability for the delay-sensitive traffic. This metric is closely tied back to the well-developed theory of effective capacity [19]. It was first introduced by $\mathrm{Wu}$ and Negi in [19] to describe the maximum arrival rate a given service process can support in order to guarantee a QoS requirement. From the definition of effective capacity, it is observed that QoS provisioning performance is related with wireless channel service rate via effective capacity. Thus, it can be employed to model cross-layer design between the physical layer system infrastructure and statistical QoS performance at the data link layer. With it, a set of statistical delay QoSdriven resource allocation policies has been addressed [20-22]. In particular, [21] investigates resource allocation including subcarrier and power allocation for LTE-A relay networks under statistical QoS constraints. With statistical QoS constraint, maximum acceptable end-to-end queue-length bound outage probability has been investigated for a three-node buffer-aided relaying network in [22].

Based on the aforementioned motivation, the current work adopts the statistical delay provisioning and effective capacity to allocate the wireless resources in a spectrumleasing based CRN. Specifically, by jointly considering the physical layer and data link layer, we will develop a crosslayer based, QoS-oriented power, and time allocation policy. In the considered CRN scenario, the secondary system helps the primary system via three-phase cooperative relaying, where both AF and DF relay protocols are discussed. Our work is motivated by the observation that cooperation model brings a waiting delay to the PUs, since SU has to alternately forward the primary traffic and send its secondary traffic on the same channel. Given the competitive and selfish properties of users, two design goals are given. On one hand, the goal of the primary link aims to minimize its expense of radio resources (i.e., the leased time). On the other hand, the object is to minimize the overall power consumption of the network. In practice, these two design objectives are all desirable for the system operators. However, they are conflicting with each other, and each focuses on only one aspect of the system. Via employing the multi-objective optimization theory, these two design objectives are realized simultaneously with statistical delay provisioning.

The main contributions of this work are summarized as follows:

\ Firstly, we formulate the resource allocation as a cross-layer optimization problem, where multiple conflicting objectives are interrelated and minimized at the same time. Specifically, by employing multi-objective optimization theory, we model the resource allocation as minimizing both the power consumption of the global network and the leased time to SU simultaneously, while fulfilling the statistical delay QoS constraints of all users. As far as we know, this is the first work that configures cooperative relaying schemes jointly with transmit powers, leased time, and statistical delay QoS requirements.

\ Secondly, via employing weighting method, the adopted multi-objective optimization problem is recast as a convex optimization one. With it, the closed-form expressions of the optimal power and time allocation strategies are derived by using the Lagrangian dual method. We show that the global optimal solution of the original problem leads to a set of Pareto-optimal resource allocation policies. That is, we obtain a set of compromises characterized by a Pareto set which provides a quantitative insight into the fundamental tradeoff between the transmit power and the leased time. Furthermore, we also analyze the impact of delay exponents on the overall performance and characterize the properties of the optimal resource allocation strategies.

- Thirdly, considering that it is hard or impossible to have a priori knowledge of the cumulative distribution function $(\mathrm{CDF})$ of the time-varying fading channels, 
we model the channel condition as a stochastic process. Based on the stochastic optimization tools [23-26], a stochastic iterative algorithm is proposed to learn the underlying channel distribution.

The rest of the paper is organized as follows. Section 2 describes the system model of concern and the delay QoS provisioning based on effective capacity. Sections 3 and 4 study the optimal resource allocation strategies such that the minimum power and leased time are consumed while fulfilling the statistical delay provisioning, when employing $\mathrm{AF}$ and DF relay protocols, respectively. In Section 5, we propose the stochastic resource allocation algorithms to approach the globally solutions. Numerical results are illustrated in Section 6, followed by the conclusions drawn in Section 7.

\section{System overview}

In the following, we detail the system model of spectrum leasing, the main system parameters, and the concept of effective capacity.

\subsection{System model}

We consider two infrastructure-based networks, where the primary network and the secondary network are located in the same area. The primary network is willing to share its licensed spectrum with the secondary network as an exchange for cooperation. Thereinto, a primary transmitter (PT) tends to communicate with primary receiver (PR) with its own spectrum and a secondary transmitter (ST) communicates with secondary receiver (SR) by using the leased spectrum. As introduced, by employing the effective capacity theory a cross-layer-based transmitter structure is formulated, in which an infinite queue (or buffer) operating in a first-in-first-out (FIFO) mode is implemented at the data link layer to store frames to be transmitted. Frames from upper layers are put into the queue. Then at the physical layer, frames from the queue are divided into bit-streams. The reverse operations are executed at the receiver side.

We assume that both the PU and SU experience independent fading, and their fading processes are stationary and ergodic with joint cumulative distribution. The channel coefficients of $\mathrm{PT} \rightarrow \mathrm{PR}, \mathrm{PT} \rightarrow \mathrm{ST}, \mathrm{ST} \rightarrow \mathrm{PR}$, and $\mathrm{ST} \rightarrow \mathrm{SR}$ links are denoted as $h_{p p}, h_{p s}, h_{s p}$, and $h_{s s}$, respectively. And the corresponding channel variance of each link is denoted by, $\sigma_{p p}^{2}, \sigma_{p s}^{2}, \sigma_{s p}^{2}, \sigma_{s s}^{2}$. Without loss of generality, we assume that all the noise terms are white Gaussian noise with zero mean and variance $\sigma_{p p}^{2}=\sigma_{p s}^{2}=\sigma_{s p}^{2}=$ $\sigma_{s s}^{2}=1$. Additionally, the wireless links are assumed to experience fading from one frame to another but remain invariant within a frame duration. Thus, the channel gains are denoted as $\gamma_{p p}=\left|h_{p p}\right|^{2}, \gamma_{p s}=\left|h_{p s}\right|^{2}, \gamma_{s p}=\left|h_{s p}\right|^{2}$, and $\gamma_{s s}=\left|h_{s s}\right|^{2}$, respectively. $\gamma$ is defined as $\gamma:=$ $\left\{\gamma_{p p}, \gamma_{p s}, \gamma_{s p}, \gamma_{s s}\right\}$. Let us denote the system's total spectral bandwidth by $B$. Suppose that perfect channel state information is always available at transmitter side. We also assume that proper channel code can always be found to commit error-free transmission.

In this paper, we study the cooperation strategy with time-division-based half duplex AF and DF relay protocols, respectively. The cooperation between PU and SU involves three phases. Phases I and II are called as the cooperative communications, whose length of time are both assumed $T_{p}$. In the phase I, the PT sends its signals while SU and PR listen. If PT transmits over his assigned time fraction $T_{p}$ with rate $\rho_{p}$ and power $\pi_{p}$, then clearly its overall transmission rate and power per spectrum sharing period are $r_{p}=\rho_{p} T_{p}$ and $p_{p}=\pi_{p} T_{p}$, respectively. In the phase II, SU forwards the received signal to PR according to AF or DF relay policy. Then PR combines signal copies from the two phases. Suppose that ST forwards the primary signals at his assigned time fraction $T_{p}$ with rate $\rho_{s p}$ and power $\pi_{s p}$, then clearly its overall transmission rate and power per spectrum sharing period are $r_{s p}=\rho_{s p} T_{p}$ and $p_{s p}=\pi_{s p} T_{p}$, respectively. In phase III, ST is rewarded the spectrum for its own transmission as a remuneration of forwarding primary signals with a dedicated time duration. We premise that the ST transmits over its spectrum sharing period $t_{s}$ with rate $\rho_{s s}$ and power $\pi_{s s}$, then clearly its overall transmission rate and power per spectrum sharing period are $r_{s}=\rho_{s s} t_{s}$ and $p_{s s}=\pi_{s s} t_{s}$, respectively. Thus, the period of cooperative spectrum sharing (i.e., phases I, II, and III) is

$$
T=T_{p}+T_{p}+t_{s} .
$$

Then with the AF relay protocol, using transmit power $\pi_{p}$ and $\pi_{s p}$, the transmission rate (bits/s/Hz) of the primary system at PR is $[27,28]$

$$
\rho_{p}=\log _{2}\left(1+\pi_{p} \gamma_{p p}+\frac{\pi_{p} \gamma_{p s} \pi_{s p} \gamma_{s p}}{1+\pi_{p} \gamma_{p s}+\pi_{s p} \gamma_{s p}}\right) .
$$

Taking into account the time fraction $T_{p}$, the service rate $^{1}$ of the primary system at PR during the cooperation communication (phases I and II) reads

$$
R_{p}^{\mathrm{AF}}=r_{p} B=T_{p} B \log _{2}\left(1+\frac{p_{p}}{T_{p}} \gamma_{p p}+\frac{\frac{p_{p}}{T_{p}} \gamma_{p s} \frac{p_{s p}}{T_{p}} \gamma_{s p}}{1+\frac{p_{p}}{T_{p}} \gamma_{p s}+\frac{p_{s p}}{T_{p}} \gamma_{s p}}\right)
$$

Similarly, with the DF relay protocol, the service rate [29] at PR during the cooperation communication follows

$$
\begin{aligned}
R_{p}^{\mathrm{DF}}= & r_{s p} B=T_{p} B \log _{2}\left(1+\gamma_{p p} \frac{p_{p}}{T_{p}}\right) \\
& +T_{p} B \log _{2}\left(1+\gamma_{s p} \frac{p_{s p}}{T_{p}}\right) .
\end{aligned}
$$


In phase III, using transmit power $\pi_{s s}$, ST can theoretically transmit with rate $(\mathrm{bits} / \mathrm{s} / \mathrm{Hz})$

$$
\rho_{s s}=\log _{2}\left(1+\pi_{s s} \gamma_{s s}\right) \text {. }
$$

Taking into account the leased time $t_{s}$, the service rate of the secondary user is

$$
R_{s s}=t_{s} B \log _{2}\left(1+\frac{p_{s s} \gamma_{s s}}{t_{s}}\right)
$$

\subsection{Statistical delay QoS guarantees and effective capacity}

As mentioned, we will introduce effective capacity to describe the system throughput with statistical delay QoS guarantees. Before going further, we would first spend some words on effective bandwidth [30] which is the dual problem of effective capacity. The stochastic behavior of a source traffic flow can be modeled asymptotically by its effective bandwidth. In particular, it is defined as the minimum service rate required by a given arrival process for which a QoS requirement is guaranteed. As its dual problem, effective capacity defines the maximum rate the channel can support while guaranteeing a given delay QoS requirement in terms of QoS exponent $\theta>0$.

In this work, by adopting effective capacity theory, the delay QoS guarantees are considered in terms of $\theta . \theta$ is a crucial parameter describing the exponential decay rate of the queue length. Specifically, based on the largedeviation theory and premise that the steady-state queue length exists, the probability of the queue length $Q(t)$ exceeding a certain threshold $x$ decays exponentially fast as $x$ increases [30], shown as

$$
-\lim _{x \rightarrow \infty} \frac{\ln (\operatorname{Pr}\{Q(\infty) \geq x\})}{x}=\theta .
$$

In (7), smaller and larger $\theta$ corresponds to slower and faster decaying rate, indicating that the system can guarantee a loose and stringent violation probability requirement, respectively.

With the QoS exponent $\theta$, the effective capacity can be described mathematically as [19]

$$
E_{C}(\theta)=-\frac{1}{\theta} \log \left(\mathbb{E}\left[e^{-\theta R}\right]\right),
$$

where $\mathbb{E}[\cdot]$ is the expectation operator, and $R$ is the maximum data rate supported by the physical layer for the traffic flow in wireless networks. With $\theta$, the probability that the steady-state delay $D(\infty)$ experienced by a traffic flow exceeds a specified delay bound $D_{\max }$ [19] that can be characterized by

$$
\operatorname{Pr}\left\{D(\infty)>D_{\max }\right\} \approx \varrho e^{-\theta E_{B} D_{\max }},
$$

where $\varrho$ denotes the non-empty probability of the buffer, and $\varrho \approx E_{B} / \mu_{C}$ and $\mu_{c} \triangleq \lim _{\theta \rightarrow 0} E_{c}(\theta)$ [20], $E_{B}$ is the effective bandwidth with QoS exponent $\theta$ of a source traffic flow. For details, readers are referred to $[19,20,30]$.
Mathematically, when $E_{C}(\theta) \geq E_{B}$ holds, the delay QoS guarantees of users can be satisfied. It serves as a reference when assigning resource at physical layer. Then in current work, in order to fulfill the statistical delay QoS requirements of PU and SU, the following constraints must be satisfied

$$
\begin{aligned}
& -\frac{1}{\theta_{p}} \log \left\{\mathbb { E } \left[e^{\left.\left.-\theta_{p} R_{p}^{A F}\right]\right\} \geq E_{p}, \quad \text { or }}\right.\right. \\
& -\frac{1}{\theta_{p}} \log \left\{\mathbb{E}\left[e^{-\theta_{p} R_{p}^{D F}}\right]\right\} \geq E_{p}, \\
& -\frac{1}{\theta_{s}} \log \left\{\mathbb{E}\left[e^{-\theta_{s} t_{s} B \log _{2}\left(1+\frac{p_{s s} \gamma_{s s}}{t_{s}}\right)}\right]\right\} \geq E_{s},
\end{aligned}
$$

where $\theta_{p}$ and $\theta_{s}$ are the delay QoS exponents of PU and SU, respectively. $E_{p}$ and $E_{s}$ denote the required effective bandwidth of the source traffic flow of the primary system and the secondary system, respectively. Considering that $\log (\cdot)$ is a monotonically increasing function and $\theta_{p}>0, \theta_{s}>0$, the above two constraints are equivalent to

$$
\mathbb{E}\left[e^{-\theta_{p} R_{p}^{A F}}\right] \leq e^{-\theta_{p} E_{p}}, \quad \text { or } \quad \mathbb{E}\left[e^{-\theta_{p} R_{p}^{D F}}\right] \leq e^{-\theta_{p} E_{p}},
$$

$$
\mathbb{E}\left[e^{-\theta_{s} t_{s} B \log _{2}\left(1+\frac{p_{s s} \gamma_{s s}}{t_{s}}\right)}\right] \leq e^{-\theta_{s} E_{s}}
$$

\section{Optimal power and time allocation with AF relay protocol}

In this paper, we focus on how to allocate the available power and time between PU and SU so as to minimize both the average sum power and the average leased time, while fulfilling the statistical delay provisioning. When employing the AF relay protocol, the optimization problem can be mathematically formulated as

$$
\begin{aligned}
& \mathbf{P 1}: \min _{\boldsymbol{p}, t_{s}} \mathbb{E}\left[p_{p}+p_{s p}+p_{s s}\right], \mathbb{E}\left[t_{s}\right], \\
& \text { s.t. } \quad \mathbb{E}\left[e^{-\theta_{p} T_{p} B \log _{2}\left(1+\frac{p_{p}}{T_{p}} \gamma_{p p}+\frac{\frac{p_{p}}{T_{p}} \gamma_{p s} \frac{p_{s p}}{T_{p}} \gamma_{s p}}{T_{p} \gamma_{p s}+\frac{p_{p s}}{T_{p}} \gamma_{s p}}\right)}\right] \leq e^{-\theta_{p} E_{p}}, \\
& \mathbb{E}\left[e^{-\theta_{s} t_{s} B \log _{2}\left(1+\frac{p_{s s} \gamma_{S s}}{t_{s}}\right)}\right] \leq e^{-\theta_{s} E_{s}},
\end{aligned}
$$

where $\boldsymbol{p}=\left\{p_{p}, p_{s p}, p_{s s}\right\}$ and $t_{s}$ are the optimization variables. Notice that, these two objectives conflict with each other. For instance, in order to shorten the leased time from the PU, SU must increase its transmit power to satisfy the delay requirements. In fact, via the concept of Pareto optimality, the tradeoff between these conflicting design objectives can be investigated. In particular, 
the adopted multi-objective optimization enables the design of a set of Pareto-optimal resource allocation strategies [31].

A simple and efficient way to solve P1 is the weighting method [32], which combines two objective functions into a single objective function with weight factors. Thus, by taking this approach, we can obtain

$$
\begin{aligned}
\mathbf{P 2}: & \min _{\boldsymbol{p}, t_{s}} \omega_{1} \mathbb{E}\left[p_{p}+p_{s p}+p_{s s}\right]+\omega_{2} \mathbb{E}\left[t_{s}\right], \\
& \text { s.t. (15), (16). }
\end{aligned}
$$

Thereinto, $\left\{\omega_{k}, k=1,2\right\}$ are the non-negative weight factors, and $\omega_{1}+\omega_{2}=1$. They denote the importance of each objective function. The optimal solutions to P2 for different values of $\omega_{k}, \forall k$, collectively form the Pareto-optimal set of P2. That is, by tuning $\omega_{k}$, we are to investigate the tradeoff between the transmit power and the leased time with statistical delay provisioning.

The underlined optimization problem P2 is a convex optimization problem, and a detailed proof is given in Appendix 1. Then we will employ the Lagrangian dual approach [33] to solve this optimization problem with given $\omega_{k}, \forall k$.

\subsection{Optimizing the dual problem}

Mathematically, by relaxing the constraints, the Lagrangian is expressed as

$$
\begin{aligned}
& L_{A F}\left(\boldsymbol{p}, t_{s}, \boldsymbol{Z}_{1}\right)=\omega_{1} \mathbb{E}\left[p_{p}+p_{s p}+p_{s s}\right]+\omega_{2} \mathbb{E}\left[t_{s}\right]+\xi\left(\mathbb{E}\left[e^{-\theta_{s} t_{s} B \log _{2}\left(1+\frac{p_{s s} \gamma_{s s}}{t_{s}}\right)}\right]\right.
\end{aligned}
$$

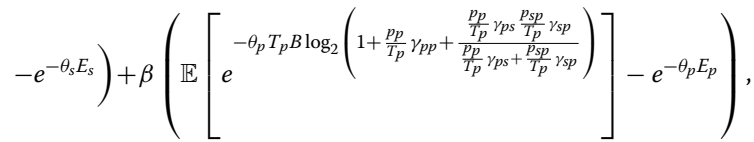

where $\beta$ and $\xi$ are two introduced Lagrangian multipliers associated with constraints. And $Z_{1}$ is defined as $Z_{1}:=\{\beta, \xi\}$. The dual function is given by

$$
D_{A F}\left(Z_{1}\right)=\min _{\boldsymbol{p}, t_{s}} L_{A F}\left(\boldsymbol{p}, t_{s}, Z_{1}\right),
$$

and the dual optimization problem can be expressed by

$$
\begin{array}{ll}
\max _{Z_{1}} & D_{A F}\left(Z_{1}\right), \\
\text { s.t. } & Z_{1} \succeq 0 .
\end{array}
$$

Due to the convexity of the original problem P2, the optimal value of the dual problem (20) coincides with that of the optimization problem P2, i.e., strong duality holds ([33], p. 226). Furthermore, since a dual function is convex by definition [33], subgradient-based iteration algorithm can be used to solve the dual problem (20) by updating $\beta, \xi$ along with appropriate directions, and it is guaranteed to converge to the optimal solution $\left\{\beta^{*}, \xi^{*}\right\}$. Mathematically, the subgradient of $D_{A F}\left(Z_{1}\right)$ follows

$\left\{\begin{array}{l}\beta[n+1]=[\beta[n]+s . \\ \left(\mathbb{E}\left[e^{\left.\left.\left.-\theta_{p} T_{p} B \log _{2}\left(1+\frac{p_{p}^{*}\left(Z_{1}\right)}{T_{p}} \gamma_{p p}+\frac{\gamma_{p s} \gamma_{s p}}{T_{p}} \cdot \frac{p_{p}^{*}\left(Z_{1}\right) p_{s p}^{*}\left(Z_{1}\right)}{p_{p}^{*}\left(Z_{1}\right) \gamma_{p s}+p_{s p}^{*}\left(Z_{1}\right) \gamma_{s p}}\right)\right]-e^{\left.-\theta_{p} E_{p}\right)}\right)\right]^{+},}\right.\right. \\ \xi[n+1]=\left[\xi[n]+s \cdot\left(\mathbb{E}\left[e^{-\theta_{s} t_{s} B \log _{2}\left(1+\frac{p_{s s}^{*}\left(Z_{1}\right) \gamma_{s s}}{t_{s}^{*}\left(Z_{1}\right)}\right)}\right]-e^{-\theta_{s} E_{s}}\right)\right]^{+},\end{array}\right.$

where $n$ is the iteration index, $s>0$ is defined as a positive stepsize, and $[\cdot]^{+}$denotes the projection onto the non-negative orthant. If the step size $s$ follows the diminishing step size policy in [34], the subgradient method above is guaranteed to converge to the optimal dual variables $Z_{1}^{*}$. The computational complexity of such update method is polynomial in the number of dual variables [33]. Moreover, $\left\{\boldsymbol{p}^{*}, t_{s}^{*}\right\}$ are the optimal solution of maximizing $L_{A F}\left(\boldsymbol{p}, t_{s}, Z_{1}\right)$ for given $Z_{1}^{*}$.

\subsection{Optimal power and time allocation policy with given Lagrangian variables}

To find the optimal $\left\{\boldsymbol{p}^{*}, t_{s}^{*}\right\}$ that minimizes $L_{A F}\left(\boldsymbol{p}, t_{s}, Z_{1}\right)$ in (19), we can equivalently solve the following problem

$$
\min _{\boldsymbol{p}, t_{s}} L_{A F}^{\prime}\left(\boldsymbol{p}, t_{s}, Z_{1}\right) \text {, }
$$

where

$$
\begin{aligned}
L_{A F}^{\prime}\left(\boldsymbol{p}, t_{s}, Z_{1}\right)= & \omega_{1}\left(p_{p}+p_{s p}+p_{s s}\right)+\omega_{2} t_{s}+\xi e^{-\theta_{s} t_{s} B \log _{2}\left(1+\frac{p_{s s} \gamma_{s s}}{t_{s}}\right)} \\
& \left.+\beta e^{-\theta_{p} T_{p} B \log _{2}\left(1+\frac{p_{p}}{T_{p}} \gamma_{p p}+\frac{\frac{p_{p}}{T_{p}} \gamma_{p s} \frac{p_{s p}}{T_{p}} \gamma_{s p}}{T_{p}} \gamma_{p s}+\frac{p_{s p}}{T_{p}} \gamma_{s p}\right.}\right)
\end{aligned}
$$

That is, the problem of minimizing $L_{A F}\left(\boldsymbol{p}, t_{s}, \boldsymbol{Z}_{1}\right)$ in (19) can be solved via decoupling the optimization problem of minimizing $L_{A F}^{\prime}\left(\boldsymbol{p}, t_{s}, \boldsymbol{Z}_{1}\right)$ across fading states. The detailed derivation of this result follows along the lines of ([35], Appendix III), which is omitted here for lack of space.

It can be easily shown that (22) is also a convex function of $\left\{\boldsymbol{p}, t_{s}\right\}$, and consequently the optimal solution can derived by applying Karush-Kuhn-Tucker (KKT) conditions. Thus, differentiating $L_{A F}^{\prime}\left(\boldsymbol{p}, t_{s}, Z_{1}\right)$ with respect to $p_{s s}$ and setting the derivative equal to zero, we obtain

$$
\omega_{1}=\frac{\xi \theta_{s} B \gamma_{s s}}{\ln 2}\left(1+\frac{p_{s s} \gamma_{s s}}{t_{s}}\right)^{-\frac{\ln 2+\theta_{s} B t_{s}}{\ln 2}} .
$$

Via solving (23), we can get the optimal value of allocated power over the $\mathrm{ST} \rightarrow \mathrm{SR}$ link with given $\xi$, shown as

$$
\frac{p_{s s}}{t_{s}}= \begin{cases}\Lambda_{0}^{\frac{\ln 2}{\theta_{s} B t_{s}+\ln 2}}\left(\frac{1}{\gamma_{s s}}\right)^{\frac{\theta_{s} B t_{s}}{\theta_{s} B t_{s}+\ln 2}}-\frac{1}{\gamma_{s s}}, & \Lambda_{0}>\frac{1}{\gamma_{s s}} \\ 0 & \Lambda_{0} \leq \frac{1}{\gamma_{s s}}\end{cases}
$$


where $\Lambda_{0}=\frac{\xi \theta_{s} B}{\omega_{1} \ln 2}$. Notice that, this power policy is the deterministic function of delay QoS exponent $\theta_{s}$ and channel fading states. Apparently, it has a similar expression with the conventional water-filling policy [36]. We take the first part of formula as the water level just as defined in water-filling policy. However, it is worth mentioning that our proposed policy (24) is different from the classical water-filling policy in that the water level in our case is a variable. It depends on delay QoS requirements $\theta_{s}$. But the water level given in water-filling policy is a constant and is not related to the system delay requirement. In the proposed policy, if there is no constraint on delay, $\theta_{s}$ will be zero and the water level in (24) will become a constant. At this moment, our policy converges to the conventional water-filling policy. From this point of view, the proposed policy is a delay QoS-based water-filling policy.

Simultaneously applying the derivative of $L^{\prime}\left(\boldsymbol{p}, t_{s}, \boldsymbol{Z}_{1}\right)$ with respect to variable $p_{s p}$ and $p_{p}$, we obtain the optimal power allocation

$$
\frac{p_{p}}{T_{p}}=\left\{\begin{array}{ll}
\chi \frac{p_{s p}}{T_{p}}, & \text { if } \frac{p_{s p}}{T_{p}}>0 \\
\left(\frac{\theta_{p} B \beta}{\ln 2 \omega_{1}}\right)^{\frac{\ln 2}{\ln 2+\theta_{p} B T_{p}}} \cdot\left(\frac{1}{\gamma_{p p}}\right)^{\frac{\theta_{p} B T_{p}}{\ln 2+\theta_{p} B T_{p}}}-\frac{1}{\gamma_{p p}}, & \text { if } \frac{p_{s p}}{T_{p}}=0
\end{array},\right.
$$

and

$$
\frac{p_{s p}}{T_{p}}= \begin{cases}\left(\frac{\chi \gamma_{p s}+\gamma_{s p}}{\chi^{2} \gamma_{p p} \gamma_{p s}+\chi \gamma_{p p} \gamma_{s p}+\chi \gamma_{p s} \gamma_{s p}}\right. & \\ \left.\cdot\left(\left(\frac{\beta \theta_{p} B}{\ln 2 \omega_{1}} \cdot \frac{\chi^{2} \gamma_{p s}^{2} \gamma_{s p}}{\left(\chi \gamma_{p s}+\gamma_{s p}\right)^{2}}\right)^{\frac{\ln 2}{\ln 2+\theta_{p} B T_{p}}}-1\right)\right)^{+}, & \text {if } \gamma_{s p}>\gamma_{p p} \\ 0 & \text { if } \gamma_{s p} \leq \gamma_{p p}\end{cases}
$$

where

$$
\chi=\frac{\sqrt{\gamma_{p p} \gamma_{s p}-\gamma_{p p} \gamma_{p s}+\gamma_{p s} \gamma_{s p}}+\gamma_{p p}}{\gamma_{s p}-\gamma_{p p}} \cdot \frac{\gamma_{s p}}{\gamma_{p s}}
$$

and $(x)^{+} \triangleq(0, x)$. Refer to Appendix 2 for the details of the proof.

It indicates from (25) and (26) that for some particular channel states, i.e., $\gamma_{s p} \leq \gamma_{p p}$, no power is assigned over the ST $\rightarrow$ PR link. In this case, direct transmission (without the help of relay) is preferred so as to save the consumed power. And the power policy of direct transmission follows the expression of delay QoS-based water-filling approach shown as in (25). Otherwise if $\gamma_{s p}>\gamma_{p p}$, both PT and ST should be assigned non-zero powers, which are related through the parameter $\chi$ illustrated in (25).

Sequentially, we will derive the optimal time allocation policy by assuming the optimal power allocation has been given. The derivative of $L^{\prime}\left(\boldsymbol{p}, t_{s}, \xi, \beta\right)$ with respect to variable $t_{s}$ follows as

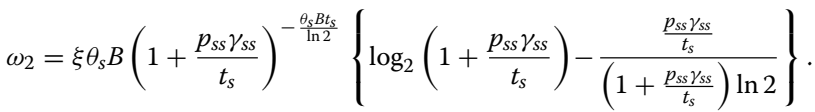

In the case of $p_{s s}>0$, substituting (24) into (28), we can get

$$
\begin{aligned}
& \frac{\omega_{2}}{\xi \theta_{s} B}\left(\Lambda_{0} \gamma_{s s}\right)^{\frac{\theta_{s} B t_{s}}{\ln 2+\theta_{s} B t_{s}}}-\frac{\ln 2}{\ln 2+\theta_{s} B t_{s}} \log _{2}\left(\Lambda_{0} \gamma_{s s}\right) \\
&+\frac{1}{\ln 2}-\frac{1}{\ln 2}\left(\Lambda_{0} \gamma_{s s}\right)^{-\frac{\ln 2}{\ln 2+\theta_{s} B t_{s}}}=0 .
\end{aligned}
$$

It is observed that (29) is an equation with one unknown variable. Thus, the optimal value of leased time for $\mathrm{SU}$ at each fading state can be obtained via solving this equation. More specifically, $t_{s}^{*}$ is the non-negative real root of (29), and it is also related with delay QoS exponent $\theta_{s}$. Till now, we have achieved the optimized jointly power and time assignment, based on the given $\boldsymbol{Z}_{1}$.

\section{Optimal power and time allocation with DF relay protocol}

In this section, we extend the proposed power and time allocation algorithm to DF cooperative communication scheme. Mathematically, the resource allocation problem of minimizing the overall transmit power and leased time while fulfilling given delay QoS requirements of PU and SU can be formulated as

$$
\begin{array}{rl}
\text { P3: } \min _{\boldsymbol{p}, t_{s}} & \mathbb{E}\left[p_{p}+p_{s p}+p_{s s}\right], \mathbb{E}\left[t_{s}\right], \\
\text { s.t. } & \mathbb{E}\left[e^{-\theta_{p} B\left[T_{p} \log _{2}\left(1+\gamma_{p p} \frac{p_{p}}{T_{p}}\right)+T_{p} \log _{2}\left(1+\gamma_{s p} \frac{p_{s p}}{T_{p}}\right)\right]}\right] \leq e^{-\theta_{p} E_{p},} \\
& \mathbb{E}\left[e^{-\theta_{s} B t_{s} \log _{2}\left(1+\frac{p_{s s} \gamma_{s s}}{t_{s}}\right)}\right] \leq e^{-\theta_{s} E_{s}}
\end{array}
$$

Problem P3 is similar to P1 in that it is also a multiobjective optimization problem. Solving this problem enables us to find the fundamental tradeoff between the transmit power of the global network and the leased time and provides a set of Pareto-optimal resource allocation policy. The same approach used to solve P1 can also be followed here. Firstly, the problem is transformed into a convex one via using the weighting method. By combining two objective functions into a single objective function with weight factors, problem P3 is transformed into (33)

$$
\begin{aligned}
\text { P4: } \min _{\boldsymbol{p}, t_{s}} \omega_{1} \mathbb{E}\left[p_{p}+p_{s p}+p_{s s}\right]+\omega_{2} \mathbb{E}\left[t_{s}\right], \\
\text { s.t. (31), (32). }
\end{aligned}
$$

By changing the weight factors, the optimal solutions to P4 can collectively form the Pareto-optimal boundary of a power-time region while employing DF relaying protocol. 


\subsection{Optimizing the dual problem}

It can be easily proved that $\mathrm{P} 4$ is convex, meaning that there exists a globally optimal solution. Similar to AF cooperative communications, we will find an analytical solution based on the Lagrangian dual approach and KKT conditions which are sufficient and necessary for this problem. Thus, by relaxing the constraints, the Lagrangian function can be formulated as

$$
\begin{aligned}
& L_{\mathrm{DF}}\left(\boldsymbol{p}, t_{s}, \boldsymbol{Z}_{2}\right)=\omega_{1} \mathbb{E}\left[p_{p}+p_{s p}+p_{s s}\right]+\omega_{2} \mathbb{E}\left[t_{s}\right] \\
& +\lambda\left(\mathbb { E } \left[e^{\left.\left.-\theta_{p} B\left[T_{p} \log _{2}\left(1+\gamma_{p p} \frac{p_{p}}{T_{p}}\right)+T_{p} \log _{2}\left(1+\gamma_{s p} \frac{p_{s p}}{T_{p}}\right)\right]\right]-e^{-\theta_{p} E_{p}}\right)}\right.\right. \\
& +\mu\left(\mathbb{E}\left[e^{-\theta_{s} B t_{s} \log _{2}\left(1+\frac{p_{s s} \gamma_{s s}}{t_{s}}\right)}\right]-e^{-\theta_{s} E_{s}}\right),
\end{aligned}
$$

where $\lambda$ and $\mu$ are Lagrangian multipliers, and $Z_{2}:=$ $\{\lambda, \mu\}$. The dual function is given by

$$
D_{\mathrm{DF}}\left(\boldsymbol{Z}_{2}\right)=\min _{\boldsymbol{p}, t_{s}} L_{\mathrm{DF}}\left(\boldsymbol{p}, t_{s}, \boldsymbol{Z}_{2}\right) \text {, }
$$

and the dual optimization problem can be expressed by

$$
\begin{aligned}
& \max _{\boldsymbol{Z}_{2}} D_{D F}\left(\boldsymbol{Z}_{2}\right), \\
& \text { s.t. } \quad \boldsymbol{Z}_{\mathbf{2}} \succeq 0 .
\end{aligned}
$$

Similarly, subgradient-based methods can be used to maximize $D_{\mathrm{DF}}\left(\boldsymbol{Z}_{2}\right)$ with global convergence. Mathematically, the subgradient of $D_{\mathrm{DF}}\left(Z_{2}\right)$ follows

$$
\left\{\begin{array}{l}
\Delta \lambda=\mathbb{E}\left[e^{\left.-\theta_{p} B T_{p}\left[\log _{2}\left(1+\gamma_{p p} \frac{p_{p}^{*}\left(Z_{2}\right)}{T_{p}}\right)+\log _{2}\left(1+\gamma_{s p} \frac{p_{s p}^{*}\left(Z_{2}\right)}{T_{p}}\right)\right]\right]-e^{-\theta_{p} E_{p}},}\right. \\
\Delta \mu=\mathbb{E}\left[e^{-\theta_{s} B t_{s}^{*} \log _{2}\left(1+\frac{p_{s s}^{*}\left(Z_{2}\right) \gamma_{s s}}{t_{s}^{*}}\right)}\right]-e^{-\theta_{s} E_{s}},
\end{array}\right.
$$

where $\left\{\boldsymbol{p}^{*}, t_{s}^{*}\right\}$ denote the optimal solution in (35) at dual point $Z_{2}^{*}$. Using the step size following the diminishing step size policy as in (21), this subgradient method above can be used to calculate the optimal $Z_{2}^{*}$ with negligible (linear) computational complexity [33].

\subsection{Optimal power and time allocation policy with given Lagrangian variables}

Following the same approach adopted for AF scenario in Section 3, decoupling the optimization problem of minimizing $L_{D F}\left(\boldsymbol{p}, t_{s}, \lambda, \mu\right)$ across fading states results in

$$
\begin{aligned}
\min _{\boldsymbol{p}, t_{s}} & \omega_{1}\left(p_{p}+p_{s p}+p_{s s}\right)+\omega_{2} t_{s} \\
& +\lambda e^{-\theta_{p} B\left[T_{p} \log _{2}\left(1+\gamma_{p p} \frac{p_{p}}{T_{p}}\right)+T_{p} \log _{2}\left(1+\gamma_{s p} \frac{p_{s p}}{T_{p}}\right)\right]} \\
& +\mu e^{-\theta_{s} B t_{s} \log _{2}\left(1+\frac{p_{s s} \gamma_{s s}}{t_{s}}\right)} .
\end{aligned}
$$

Evidently, the problem in (38) is convex and the corresponding KKT optimization conditions hold. Differentiating (38) with respect to $p_{p}, p_{s s}, p_{s p}$, respectively, and equating to zero results in

$\omega_{1}=\frac{\mu \theta_{s} B \gamma_{s s}}{\ln 2}\left(1+\frac{p_{s s} \gamma_{s s}}{t_{s}}\right)^{-\frac{\ln 2+\theta_{s} B t_{s}}{\ln 2}}$,
$\omega_{1}=\frac{\lambda \theta_{p} B \gamma_{s p}}{\ln 2}\left(1+\frac{p_{p} \gamma_{p p}}{T_{p}}\right)^{-\frac{\theta_{p} B T_{p}}{\ln 2}} \cdot\left(1+\frac{p_{s p} \gamma_{s p}}{T_{p}}\right)^{-\frac{\ln 2+\theta_{p} B T_{p}}{\ln 2}}$,

$\omega_{1}=\frac{\lambda \theta_{p} B \gamma_{p p}}{\ln 2}\left(1+\frac{p_{p} \gamma_{p p}}{T_{p}}\right)^{-\frac{\theta_{p} B T_{p}+\ln 2}{\ln 2}} \cdot\left(1+\frac{p_{s p} \gamma_{s p}}{T_{p}}\right)^{-\frac{\theta_{p} B T_{p}}{\ln 2}}$.

Simultaneously solving (39), (40), and (41), the optimal value of allocated power over the $\mathrm{ST} \rightarrow \mathrm{SR}, \mathrm{PT} \rightarrow \mathrm{ST}$, and $\mathrm{ST} \rightarrow \mathrm{PR}$ links can be obtained as

$\frac{p_{s s}}{t_{s}}=\left\{\begin{array}{ll}\Gamma^{\frac{\ln 2}{\theta_{s} B t_{s}+\ln 2}}\left(\frac{1}{\gamma_{s s}}\right)^{\frac{\theta_{s} B t_{s}}{\theta_{s} B t_{s}+\ln 2}}-\frac{1}{\gamma_{s s}}, & \Gamma \geq 1 / \gamma_{s s} \\ 0 & \Gamma<1 / \gamma_{s s}\end{array}\right.$,

$\frac{p_{p}}{T_{p}}=\left\{\begin{array}{ll}\Psi^{\frac{\ln 2}{\ln 2+2 \theta_{p} B T_{p}}} \cdot\left(\frac{1}{\gamma_{p p} \gamma_{s p}}\right)^{\frac{\theta_{p} B T_{p}}{\ln 2+2 \theta_{p} B T_{p}}}-\frac{1}{\gamma_{p p}}, & \Psi \geq \Psi_{0} \\ 0 & \Psi<\Psi_{0}\end{array}\right.$,

$\frac{p_{s p}}{T_{p}}=\left\{\begin{array}{ll}\frac{\ln 2}{\Psi^{\ln 2+2 \theta_{p} B T_{p}}} \cdot\left(\frac{1}{\gamma_{p p} \gamma_{s p}}\right)^{\frac{\theta_{p} B T_{p}}{\ln 2+2 \theta_{p} B T_{p}}}-\frac{1}{\gamma_{s p}}, & \Psi \geq \Psi_{0} \\ 0 & \Psi<\Psi_{0}\end{array}\right.$,

where $\Psi_{0}=\left(\frac{1}{\gamma_{p p}}\right)^{\frac{\ln 2+\theta_{p} B T_{p}}{\ln 2}} \cdot \gamma_{s p}^{\frac{\theta_{p} B T_{p}}{\ln 2}}, \Gamma=\mu \theta_{s} B /\left(\omega_{1} \ln 2\right)$, $\Psi=\theta_{p} B \lambda /\left(\omega_{1} \ln 2\right)$. The power policies defined in (42), (43), and (44) have been shown to be delay QoS-based water-filling policies. In particular, the water level of $p_{s s} / t_{s}$ depends explicitly on $\theta_{s}$, while the water levels of the power policy associated with PU, i.e., $p_{s p} / T_{p}, p_{p} / T_{p}$, are related with $\theta_{p}$. Moreover, it is interesting to see that the water levels of $p_{p} / T_{p}$ and $p_{s p} / T_{p}$ are the same and differ from that of $p_{s s} / t_{s}$. From this point of view, the power allocation policies when adopting DF relay protocol is multi-level QoS-based water-filling policies.

Again, differentiating the Lagrangian function (38) with respect to $t_{s}$ and equating to zero results in

$$
\begin{aligned}
\omega_{2}= & \mu \theta_{s} B\left(1+\frac{p_{s s} \gamma_{s s}}{t_{s}}\right)^{-\frac{\theta_{s} B t_{s}}{\ln 2}} \\
& \cdot\left\{\log _{2}\left(1+\frac{p_{s s} \gamma_{s s}}{t_{s}}\right)-\frac{\frac{p_{s s} \gamma_{s s}}{t_{s}}}{\left(1+\frac{p_{s s} \gamma_{s s}}{t_{s}}\right) \ln 2}\right\} .
\end{aligned}
$$

Substituting (42) into (45) in the case of $p_{s s}>0$ results in

$$
\begin{aligned}
\frac{\omega_{2}}{\mu \theta_{s} B}\left(\Gamma \gamma_{s s}\right)^{\frac{\theta_{s} B t_{s}}{\ln 2+\theta_{s} B t_{s}}} & +\frac{1}{\ln 2}-\frac{1}{\ln 2}\left(\Gamma \gamma_{s s}\right)^{-\frac{\ln 2}{\ln 2+\theta_{s} B t_{s}}} \\
& -\frac{\ln 2}{\ln 2+\theta_{s} B t_{s}} \log _{2}\left(\Gamma \gamma_{s s}\right)=0 .
\end{aligned}
$$


Via solving the Eq. (46), we can get the optimal value of leased time for SU at each fading state.

\section{The stochastic resource allocation algorithm}

To solve the dual problem (20) with AF relaying strategy, we need the explicit knowledge of fading channel CDF to evaluate the expected values involved in the subgradient algorithm (21). But in some practical mobile environments, it is infeasible or impossible to obtain the CDF of the fading channels. Consequently, the power and time allocation problem of operating without the knowledge of channel CDF should be tackled urgently. As it turns out, this problem can be solved via employing the stochastic optimization theory [24]. Instead of the need of CDF in (21), we drop the expectation operators $\mathbb{E}$ from the subgradient in (21) and put forward the following iterations based on one fading realization $\gamma[n]$ per iteration $n$

$$
\left\{\begin{array}{l}
\hat{\beta}[n+1]=\left[\hat{\beta}[n]+s \cdot\left(-e^{-\theta_{p} E_{p}}\right.\right. \\
\left.+e^{\left.-\theta_{p} B T_{p} \log _{2}\left(1+\frac{p_{p}^{*}\left(\gamma[n], \hat{\left.Z_{1}[n]\right)}\right.}{T_{p}} \gamma_{p p}+\frac{\gamma_{p s} \gamma_{s p}}{T_{p}} \frac{p_{p}^{*}\left(\gamma[n], \hat{Z}_{1}[n]\right) p_{s p}^{*}\left(\gamma[n], \hat{Z_{1}}[n]\right)}{p_{p}^{*}\left(\gamma[n], \hat{Z}_{1}[n]\right) \gamma_{p s}+p_{s p}^{*}\left(\gamma[n], \hat{Z}_{1}[n]\right)}\right)\right)}\right]^{+} \\
\hat{\xi}[t+1]=\left[\hat{\xi}[t]+s \cdot\left(e^{-\theta_{s} B t_{s} \log _{2}\left(1+\frac{p_{s s}^{*}\left(\gamma[n], \hat{Z_{1}}[n]\right) \gamma_{s s}}{t_{s}}\right)}-e^{-\theta_{s} E_{s}}\right)\right]^{+} .
\end{array}\right.
$$

And hats are used to underscore that these iterations are stochastic estimate instead of average values of those in (21). It only requires the fading state of the channels at the current iteration, which can be easily measured. The convergence of the stochastic subgradient iteration can be guaranteed by the following lemma.

Lemma 1 IfCDF of the ergodic fading channels is continuous, when (21) and (47) are respectively initialized with $\beta[0]=\hat{\beta}[0], \xi[0]=\hat{\xi}[0]$, respectively, then

$$
|\hat{\beta}[t]-\beta[t]| \leq \delta_{T}(s), \quad|\hat{\xi}[t]-\xi[t]| \leq \delta_{T}(s), \quad w \cdot p .1
$$

works over time interval $T$ with the constant $\delta_{T}(s) \rightarrow 0$ as the stepsize $s \rightarrow 0$.

The proof can be derived along the similar lines to those in ([37], Theorem 9.1), which is omitted here. The Lemma 1 implies the iteration of (47) converges to the optimal $\left\{\beta^{*}, \xi^{*}\right\}$ with probability 1 as stepsize $s \rightarrow 0$. Hence, the proposed stochastic policy is capable of iteratively finding the optimal $\left\{\beta^{*}, \xi^{*}\right\}$. Thus, the multiobjective resource allocation with guarantees on the effective capacity constraints is obtained, even when the fading channel distribution is unknown a priori. To go further, with (47), a stochastic power and time allocation algorithm with negligible (linear) computational complexity can be brought forward to approach the global solution, shown in Algorithm 1.

Algorithm 1 Stochastic Subgradient Iteration algorithm

Initialization: set $t=0$ and $\hat{\beta}[0]$ and $\hat{\xi}[0]$ equal to some non-negative value.

Repeat: With $\hat{\beta}[n], \hat{\xi}[n]$ available per slot $n$, allocate the power and time according to (24)-(29) between PU and SU, and update $\hat{\beta}[n+1]$ and $\hat{\xi}[n+1]$ by using (47).

Until: Required precision is satisfied.

Output: $\hat{\beta}[n]=\beta^{*}, \hat{\xi}[n]=\xi^{*}$.

To apply our stochastic resource allocation scheme to the spectrum-leasing based CRN, we can implement it in a distributed manner. The execution of the distributed iterative algorithm is illustrated as follows. Firstly, system performs some initialization such as setting $\beta[0]$ and $\xi[0]$ to some non-negative values at PT and ST, respectively. In time slot $n$, ST adjusts the leased time and the transmit power over ST $\rightarrow$ SR link according to the calculated values $t_{s}^{*}(\xi[n]), p_{s s}^{*}(\xi[n])$, and computes its power $p_{s p}^{*}(\beta[n])$ over ST $\rightarrow$ PR link which is then fed back to PT. Meanwhile, PT calculates its power value of $p_{p}^{*}(\beta[n])$. With $p_{p}^{*}(\beta[n]), p_{s p}^{*}(\beta[n])$ available, PT updates $\beta[n+1]$, which is then delivered to ST. Simultaneously, using values of $p_{s s}^{*}(\xi[n]), t_{s}^{*}(\xi[n])$, ST node updates $\xi[n+1]$. Similarly, in time slot, $n+1 \mathrm{PT}$ and ST work together well to calculate the corresponding power and time values and update the Lagrange multipliers. Eventually, the dual variable $\beta[n], \xi[n]$ will converge to the global solution $\beta^{*}, \xi^{*}$. Simultaneously, thanks to zero duality gap, the optimal solutions $p_{s s}^{*}(\xi[n]), p_{p}^{*}(\beta[n]), p_{s p}^{*}(\beta[n])$ will also converge to the globally optimal variables $p_{s s}^{*}, p_{p}^{*}, p_{s p}^{*}$.

Similarly, the stochastic resource allocation policy can also be proposed when employing DF relaying strategy. A stochastic subgradient iteration algorithm based on per slot fading realization is shown as

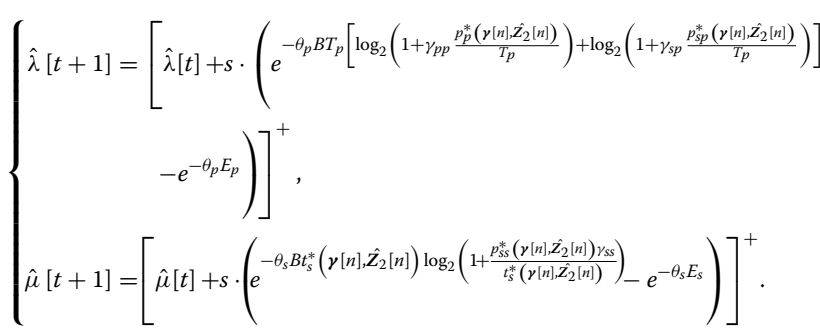

Lemma 1 holds true for $\hat{\lambda}, \hat{\mu}$, which can guarantee the convergence of this iteration. Furthermore, similar 
stochastic subgradient iteration algorithm as Algorithm 1 can also be proposed.

\section{Numerical results}

In this section, we evaluate the performance of the proposed algorithms and the tradeoff between the consumed sum power and leased time by simulations. Throughout our simulation, we consider a CRN with one pair of primary transceiver and one pair of secondary transceiver, which operate in time-slotted mode. The channel gains $\gamma_{p p}, \gamma_{p s}, \gamma_{s p}$, and $\gamma_{s s}$ undergo identical Rayleigh fading independently, and the average channel gains of all links are the same, which are set to 3 . We assume the system bandwidth is $B=1 \mathrm{kHz}$ and the duration of the cooperative communication is $1 \mathrm{~ms}$, i.e., $T_{p}=0.5 \mathrm{~ms}$. Furthermore, we assume that all users hold still, and the arrival rate of the source traffic flow is assumed to be constant. In the simulations, we set $E_{p}=1$ and $E_{s}=1$. To facilitate discussion of the impact of the weight factor on the overall transmission power and leased time, five sets of weight factor are supposed as following, $\left\{\omega_{1}=0.1, \omega_{2}=\right.$ $0.9\}$ which is labeled as w1, $\left\{\omega_{1}=0.3, \omega_{2}=0.7\right\}$ labeled as w2, $\left\{\omega_{1}=0.5, \omega_{2}=0.5\right\}$ labeled as w3, $\left\{\omega_{1}=0.7, \omega_{2}=\right.$ $0.3\}$ labeled as w4, and $\left\{\omega_{1}=0.9, \omega_{2}=0.1\right\}$ labeled as w5. More detailed parameters will accompany with results in figures to be shown.

Figure 1 shows the tradeoff regions achieved by the proposed optimal resource allocation scheme when employing AF and DF relay protocols, respectively. The Pareto boundary of the region in Fig. 1a, b is obtained by solving problems $\mathrm{P} 1$ and $\mathrm{P} 3$ via varying the values of $\omega_{k}, k \in\{1,2\}$. For a given delay QoS exponent, when $\omega_{1}$ is set to a small value, e.g., $\omega_{1}<0.3$, the Pareto boundary of the region is very sensitive to the leased time. In such scenario, the system design objective attaches importance to the leased time, and its target is to shorten the leased time at the expense of the overall power consumption. However, for higher values of $\omega_{1}$, the sensitivity of the tradeoff region is relatively dependent of the consumed power. In this case, the leased time explosively increases, while the consumed power would be inclined to a constant, e.g., 3 when $\theta_{p}=1, \theta_{s}=1$. It is noted that as $\theta_{s}$ varies from 5 to 1 , the Pareto region is narrowed. This verifies that the consumed wireless resources (i.e., power and time) decrease with the delay QoS exponents. Comparing Fig. 1a with Fig. 1b, it is no doubt that they two perform similarly in achieving the tradeoff region. The difference lies mainly in the size of the tradeoff region, resulting from the different relay protocols.

Next, we further investigate the impact of the weight factor on the consumed sum power and the average leased time. Numerical results are plotted in Figs. 2 and 3 for $\theta_{p}=\theta_{s}=1$ when $\mathrm{AF}$ and $\mathrm{DF}$ relay protocols are employed, respectively. In Figs. 2 and 3, the legend of PS represents the average power allocated over the $\mathrm{PT} \rightarrow \mathrm{ST}$ link, SP denotes the average power allocated over the $\mathrm{ST} \rightarrow \mathrm{PR}$ link, and SS denotes the average power allocated over the ST $\rightarrow$ SR link. As shown in Figs. 2 and 3, the average consumed power and average leased time simultaneously varies with the weight factors for given QoS requirements. In particular, with the increase of $\omega_{1}$, the consumed power decreases, but the average leased time synchronously becomes larger, and vice versa. This implies that the consumed power of the overall network
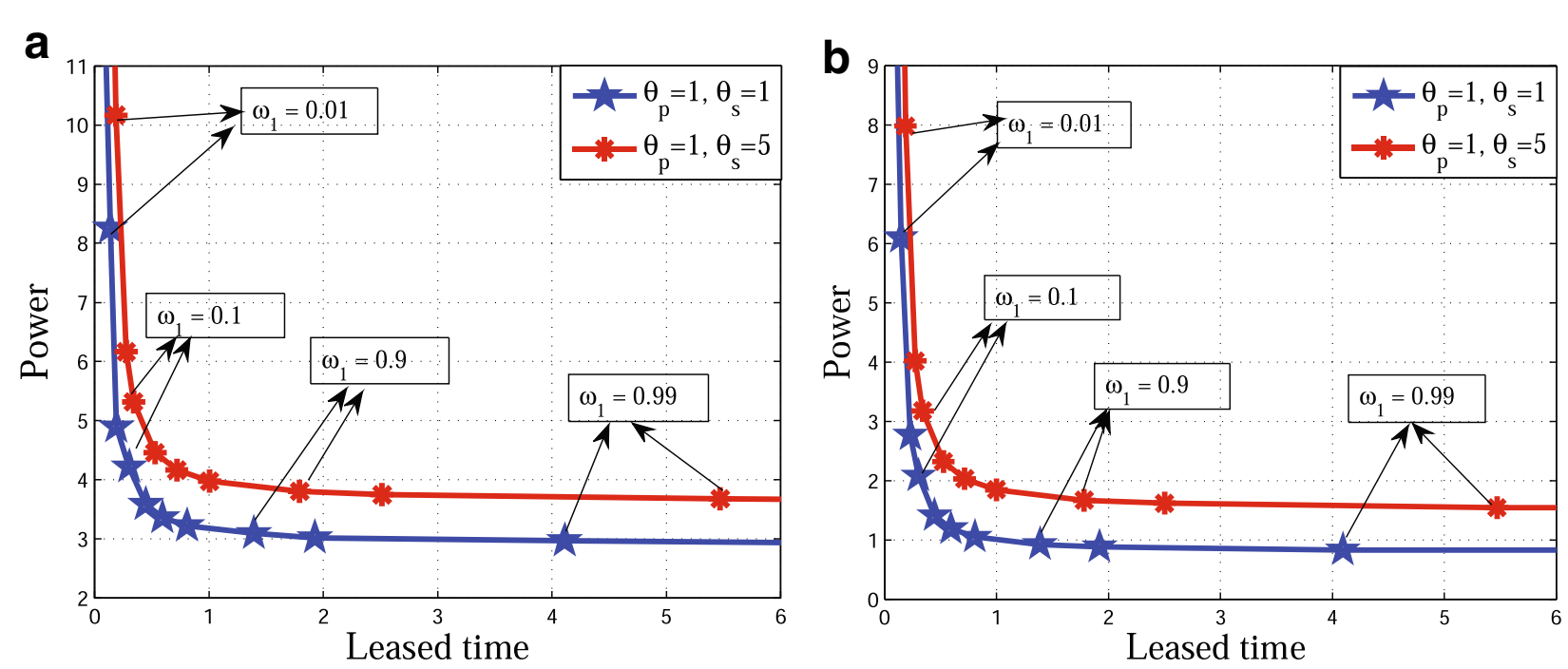

Fig. 1 System design objective tradeoff regions achieved by the proposed optimal resource allocation. a When employing AF relaying protocol. b When employing DF relaying protocol 


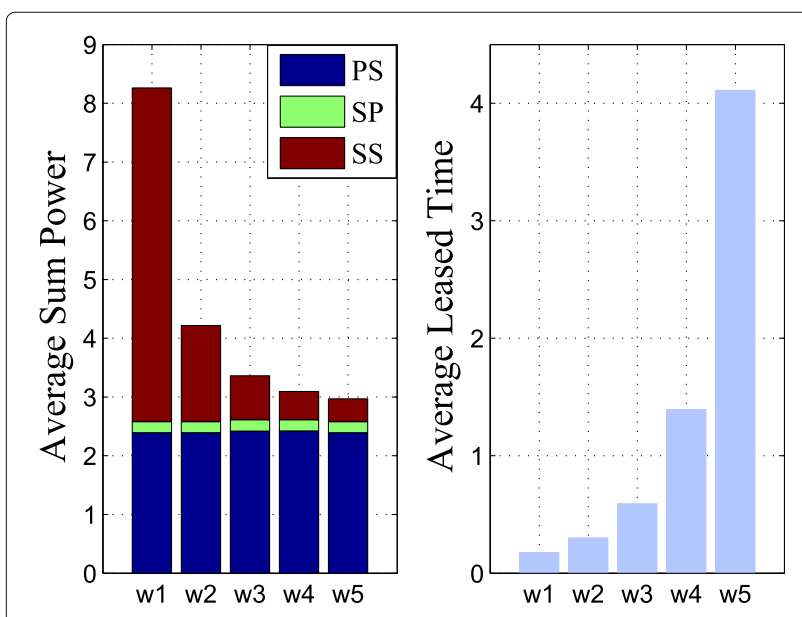

Fig. 2 The average sum power (left) and average leased time (right) with AF relay protocol. $\theta_{p}=\theta_{s}$

can be reduced via adjusting the weight factor at the expense of prolonging the leased time. Such behavior of the power and time allocation curves further characterizes the tradeoff between the transmit power and the leased time. Moreover, we can observe from Fig. 3 that due to the same fading channels and the same water level, the average transmit power of PU and that of ST forwarding the primary signals are the same when adopting the DF relay protocol. Nevertheless, this conclusion does apply to the AF relay networks, as shown in Fig. 2. This observation is coherent with the theoretical analysis made in (43), (44), and (25). Moreover, it is interesting to note that the power allocation policies in (25), (26), (43), and (44) depend on $\omega_{1}$. However, from Figs. 2 and 3, we can see that power values of PS and SP almost do not change with the weight factor. That is, the weight factor $\omega_{1}$ has little effect on these power values, which is different from the behavior of SS.

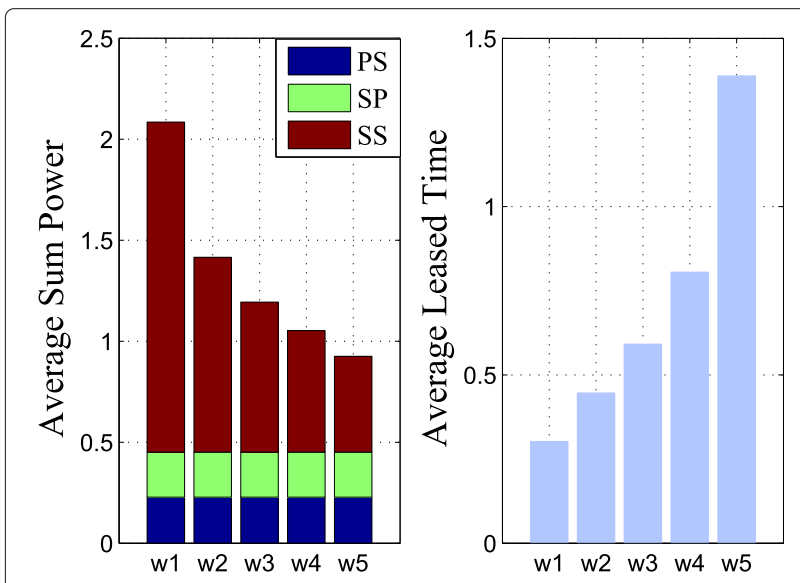

Fig. 3 The average sum power (left) and average leased time (right) with DF relay protocol. $\theta_{p}=\theta_{s}$
This is because the duration of the cooperative communications is fixed to 1 and has nothing with the weight factor.

To gauge the performance of the proposed algorithms, we compare them with other power and time allocation policies. With an equally divided time allocation, i.e., $t_{s}=$ $T_{p}=0.5 \mathrm{~ms}$, one scheme is derived from the overall average power minimization problem. It is named equal time policy. Another one is min-power policy, whose leased time is equal to the average leased time of the optimal algorithms, and its power allocation minimizes the overall average power. Figures 4 and 5 plot the average sum power and leased time against the delay QoS exponent $\theta$ for different resource allocation with AF relay protocol, when the weight factor is $\left\{\omega_{1}=0.5, \omega_{2}=0.5\right\}$ and $\left\{\omega_{1}=\right.$ $\left.0.8, \omega_{2}=0.2\right\}$, respectively. The optimal policy in Figs. 4 and 5 is the resource allocation scheme whose subgradient iterative is based on the expected values in (21), and the stochastic policy is obtained by employing the stochastic subgradient iteration algorithm proposed in Section 5. It can be observed that by carrying out the optimal policy, the average sum power is monotonically increasing with respect to QoS exponent $\theta$. This demonstrates that more power must be consumed in order to guarantee the more strict QoS requirements. Interestingly, the average leased time may not comply with this rule. In particular, for small $\theta_{s}$, the leased time increases with the QoS exponent $\theta_{s}$. However, the increasing velocity of the leased time reduces or even the leased time decreases with $\theta_{s}$. This implies that for some given weight factors and relay protocols, larger transmission rate is a must to guarantee the more stringent QoS requirements, inducing the larger ratio of the power to leased time.

As expected, the optimal policy demonstrates the same performance with the stochastic policy. This verifies that the proposed stochastic scheme can learn the channel fading knowledge on the fly and can approach the optimal policy. Remarkably, as shown in Figs. 4 and 5, the proposed policy always achieves the minimum power consumption among all control policies. In fact, in the proposed optimal scheme, both the transmit power and the leased time are jointly optimized for performing resource allocation. In contrast, the min-power policy and equal time policy are done without jointly optimization, leading to fewer degrees of freedom for resource allocation. Interestingly, the min-power performs close to the curve achieved by the optimal resource allocation scheme in loose delay QoS requirements. This observation indicates that as QoS requirement becomes loose, the leased time allocation plays only a small part in achieving the optimization objectives. As the QoS requirement getting strict, the joint power and time policy considering QoS requirement shows its advantage in power saving. And the advantage is getting larger as the requirement on delay QoS becomes more strict. Results 

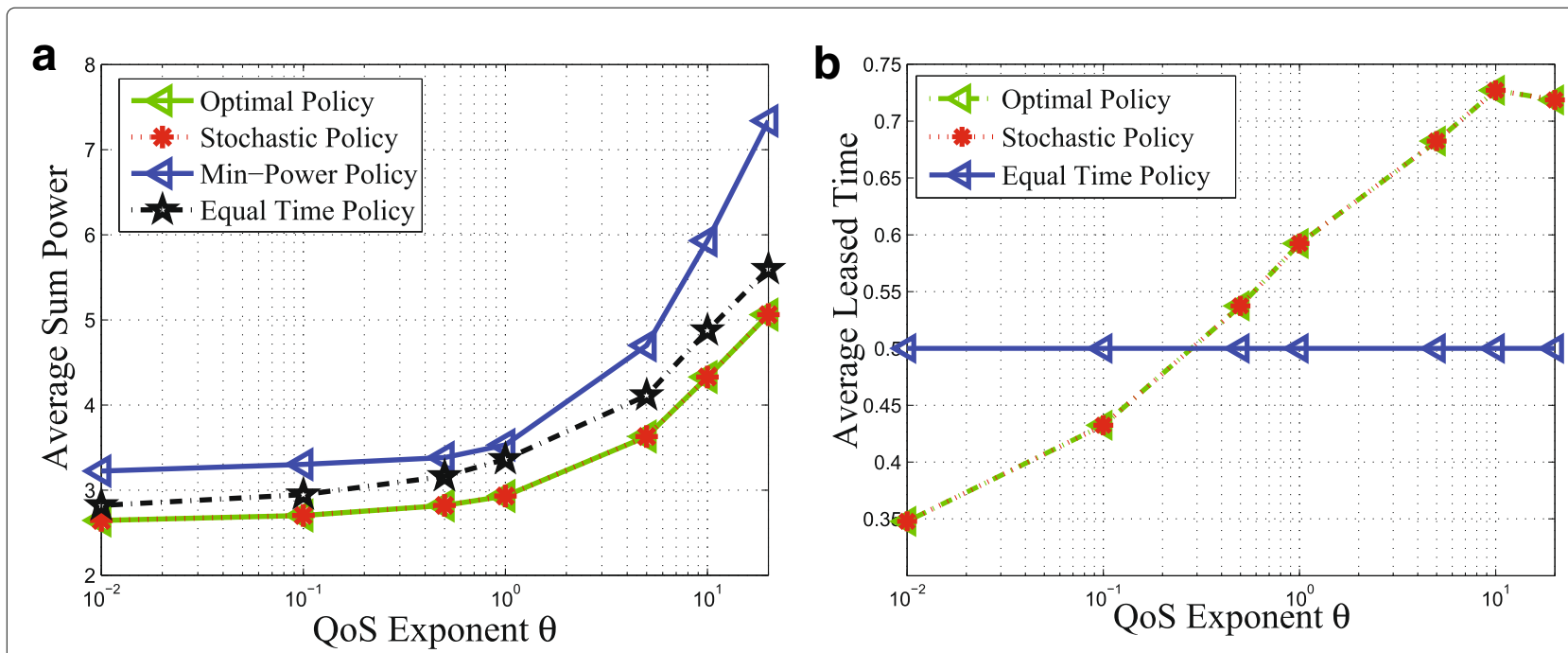

Fig. 4 a, b Average sum power and leased time versus delay QoS exponent $\theta$ with AF relay protocol when $\left\{\omega_{1}=0.5, \omega_{2}=0.5\right\}$ is adopted

when DF relay protocol is employed are also given in the case of $\left\{\omega_{1}=0.5, \omega_{2}=0.5\right\}$ and $\left\{\omega_{1}=0.8, \omega_{2}=0.2\right\}$, as shown in Figs. 6 and 7, respectively. Similar conclusions can be made that the proposed policies outperform other control policies in power saving even with loose delay QoS requirements. In summary, from Fig. 4 to Fig. 7, we can observe that the QoS requirement variations have significant impact on the effective capacity, and thus on the resource allocation of CRN.

\section{Conclusions}

In this paper, we have studied the resource allocation for spectrum-leasing CRN with effective capacity-based delay provisioning for delay-sensitive traffic. In such network, the secondary system can have the opportunities to access the licensed spectrum by employing AF/DF relay protocols to assist the primary data transmission. By integrating the multi-objective optimization theory and the concept of the effective capacity, we have formulated two optimization problems aiming to minimizing both the overall power consumption and the average leased time while fulfilling statistical delay provisioning. To solve the established problems, we resort to the weighting method that yields a convex optimization one. Then by the Lagrangian dual method, the closed-form expressions of the optimal power and time allocation strategy have been derived given the underlying statistical delay QoS constraint. We also analyze the fundamental tradeoff between the
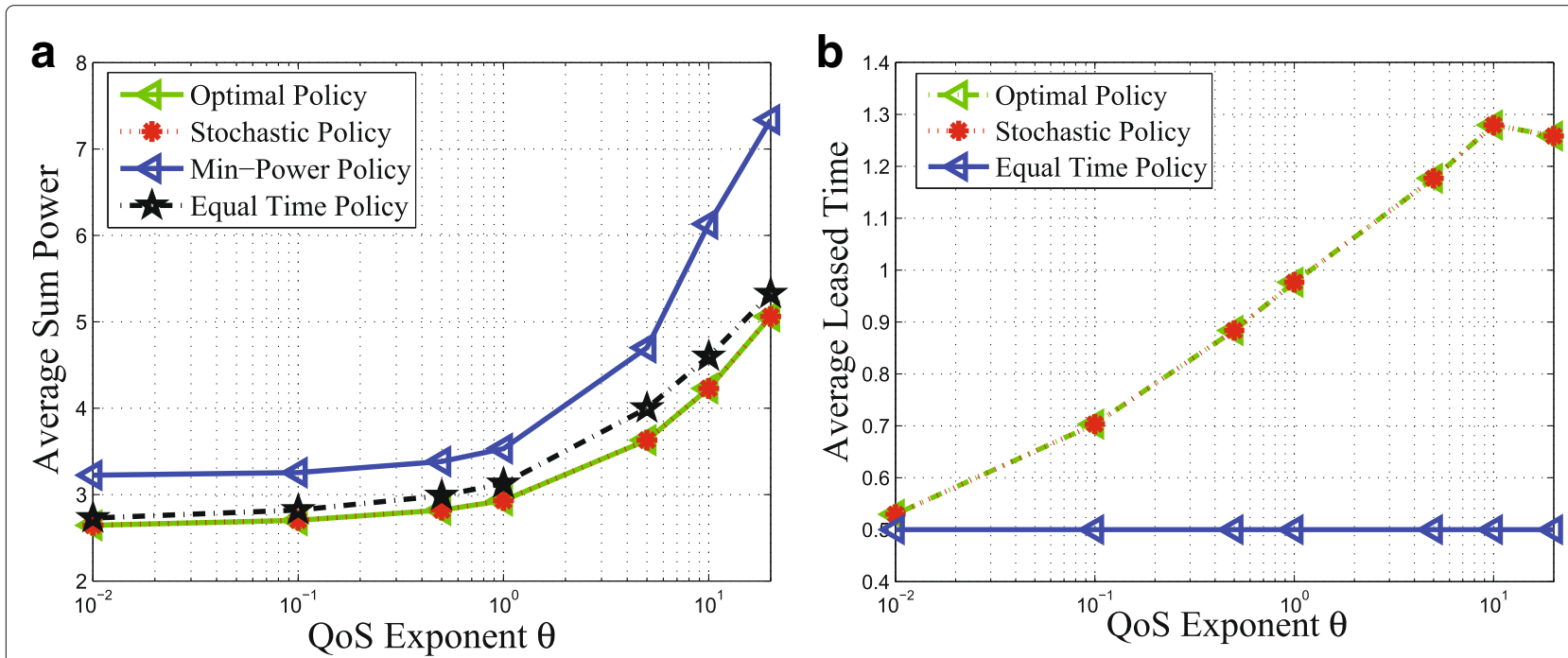

Fig. 5 a, b Average sum power and leased time versus delay QoS exponent $\theta$ with AF relay protocol when $\left\{\omega_{1}=0.8, \omega_{2}=0.2\right\}$ is adopted 

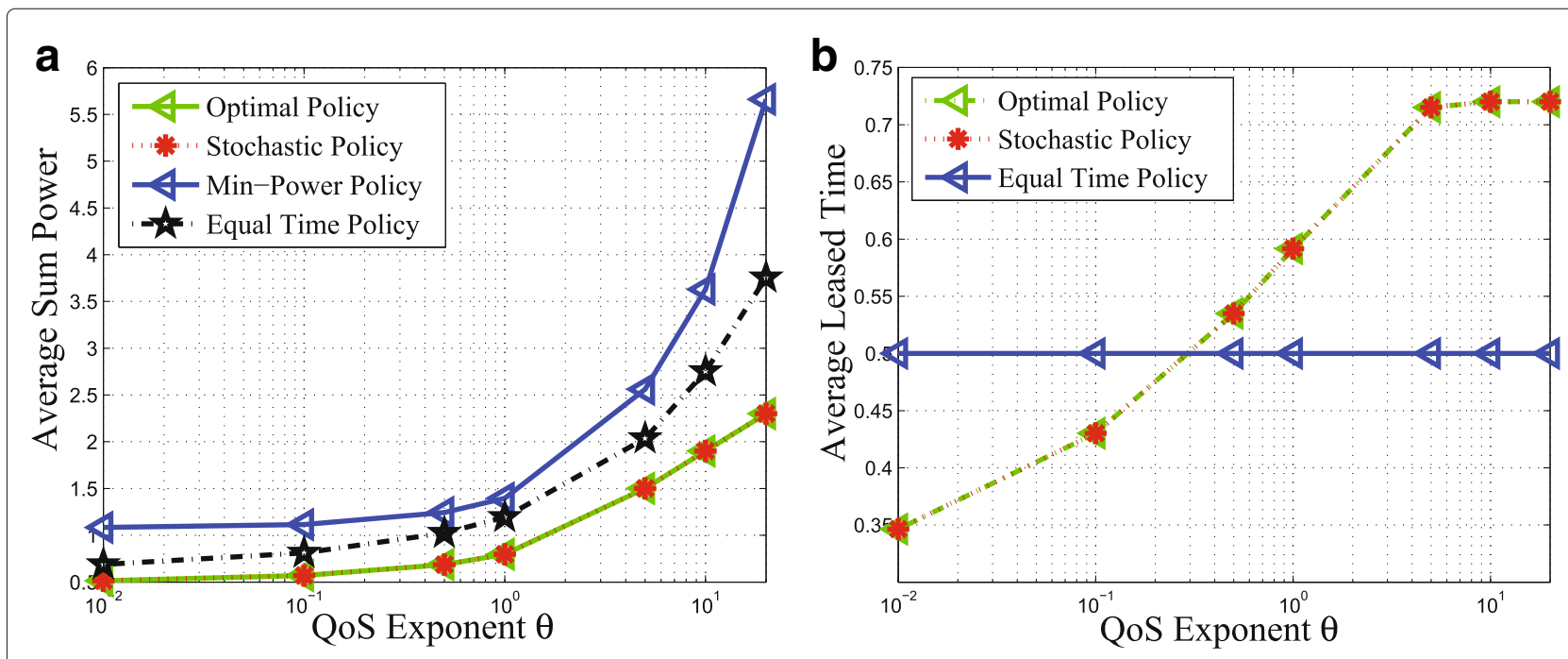

Fig. 6 a, b Average sum power and leased time versus delay QoS exponent $\theta$ with DF relay protocol when $\left\{\omega_{1}=0.5, \omega_{2}=0.5\right\}$ is adopted

transmit power and the leased time via a Pareto set, the impact of delay exponents on the overall performance, and characterize the properties of the optimal resource allocation strategies. Furthermore, we have presented stochastic resource allocation schemes that can learn the statistics of the fading channels and adaptively approach the optimal strategies on the fly. The numerical results for Rayleigh fading channels demonstrate that our proposed policies exhibit excellent performance compared with min-power policy and equal time policy.

\section{Appendix}

\section{Proof the convexity of P2}

Firstly, the objective function in P2 is convex given that $\omega_{1}\left(p_{p}+p_{s p}+p_{s s}\right)+\omega_{2} t_{s}$ is linear with respect to $p_{p}, p_{s p}, p_{s s}, t_{s}$ and that the integral preserves convexity. Then, we will demonstrate the constraint functions are all convex, guaranteeing that the feasible set of this optimization problem is convex. By evaluating the Hessian matrix of $f\left(p_{p}, p_{s p}\right)=1+\frac{p_{p}}{T_{p}} \gamma_{p p}+\frac{\frac{p_{p}}{T_{p}} \gamma_{p s} \frac{p_{s p}}{p_{p}} \gamma_{s p}}{\frac{p_{p}}{T_{p}} \gamma_{p s}+\frac{p_{s p}}{T_{p}} \gamma_{s p}}$ at $p_{p}$ and $p_{s p}$, we can prove that $f\left(p_{p}, p_{s p}\right)$ is concave. Thus, the $\log _{2}\left(f\left(p_{p}, p_{s p}\right)\right)$ is concave as $\log (\cdot)$ function can preserve concavity. Considering that exponential function $\exp (\cdot)$ and the integral preserve convexity, the first constraint function in $\mathrm{P} 2$ is convex. Given a convex function $f(x)$, its perspective $g(x, t)=t f(x / t)$ is also convex for $t>0$ [33]. For this reason, $-t_{s} \log _{2}\left(1+\frac{p_{s s} \gamma_{s s}}{t_{s}}\right)$ is the convex function of $\left(p_{s s}, t_{s}\right)$. Then, the second constraint function is convex given that exponential function $\exp (\cdot)$ and the
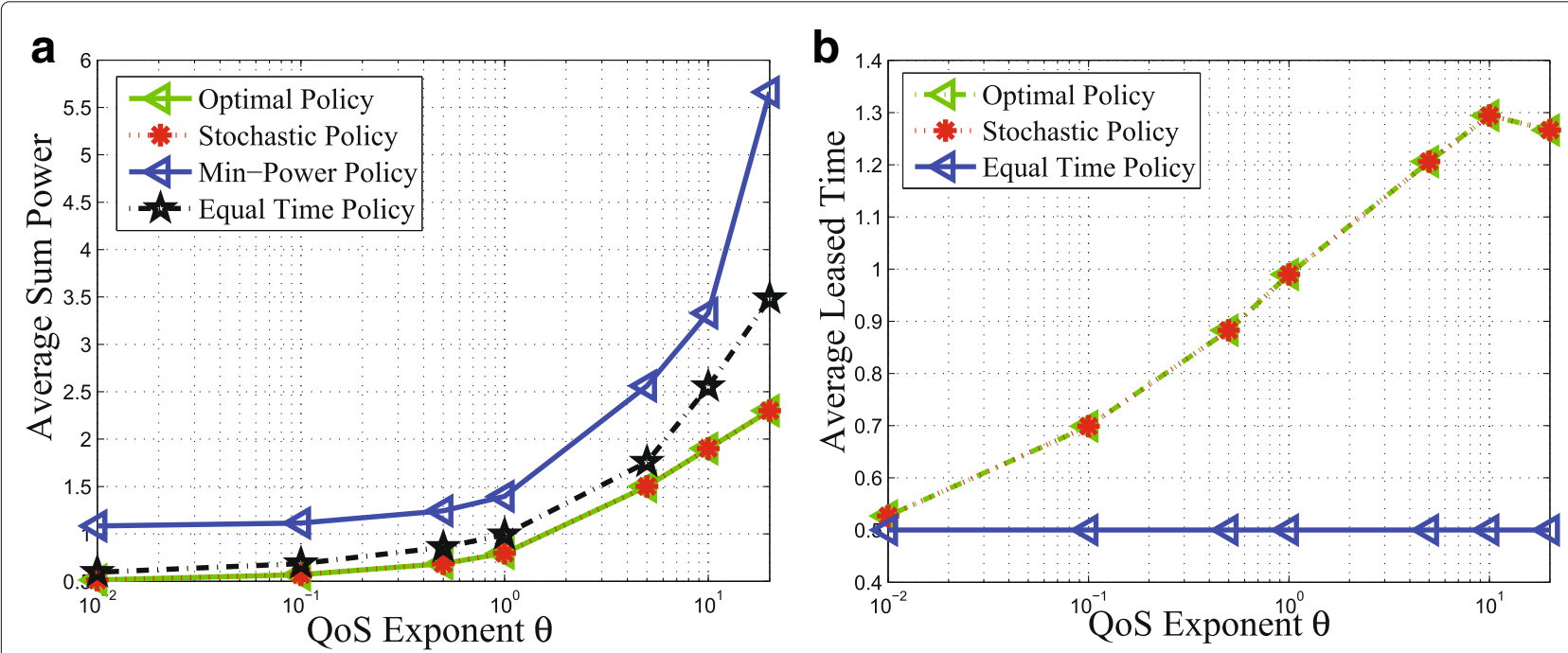

Fig. 7 a, b Average sum power and leased time versus delay QoS exponent $\theta$ with DF relay protocol when $\left\{\omega_{1}=0.8, \omega_{2}=0.2\right\}$ is adopted 
integral preserves convexity. Therefore, the problem in P2 is a convex optimization problem and there exists a unique optimal solution.

\section{Proof of optimal solution in (25) and (26)}

The derivative of $L_{A F}^{\prime}\left(\boldsymbol{p}, t_{s}, Z_{1}\right)$ with respect to variable $p_{s p}$ and $p_{p}$ is given by

$$
\begin{aligned}
\frac{\partial L_{A F}^{\prime}\left(\boldsymbol{p}, t_{s}, Z_{1}\right)}{\partial p_{s p}}= & \omega_{1}-\frac{\theta_{p} T_{p} \beta}{\ln 2}\left(1+\frac{p_{p}}{T_{p}} \gamma_{p p}+\frac{\frac{p_{p}}{T_{p}} \gamma_{p s} \frac{p_{s p}}{T_{p}} \gamma_{s p}}{\frac{p_{p}}{T_{p}} \gamma_{p s}+\frac{p_{s p}}{T_{p}} \gamma_{s p}}\right)^{-1-\frac{p_{p} T_{p}}{\ln 2}} \\
& \cdot \frac{\frac{p_{p}^{2} \gamma_{p s}^{2} \gamma_{s p}}{T_{p}^{3}}}{\left(\frac{p_{p}}{T_{p}} \gamma_{p s}+\frac{p_{s p}}{T_{p}} \gamma_{s p}\right)^{2}}, \\
\frac{\partial L_{A F}^{\prime}\left(\boldsymbol{p}, t_{s}, \boldsymbol{Z}_{1}\right)}{\partial p_{p}}= & \omega_{1}-\frac{\theta_{p} T_{p} \beta}{\ln 2}\left(1+\frac{p_{p}}{T_{p}} \gamma_{p p}+\frac{\frac{p_{p}}{T_{p}}}{\frac{p_{p}}{T_{p}} \gamma_{p s}+\frac{p_{s p}}{T_{p}} \gamma_{s p}} \gamma_{s p} \gamma_{s p}\right)^{-1-\frac{\theta_{p} T_{p}}{\ln 2}} \\
& \cdot\left(\frac{\gamma_{p p}}{T_{p}}+\frac{\frac{p_{s p}^{2} \gamma_{p s} \gamma_{s p}^{2}}{T_{p}^{3}}}{\left(\frac{p_{p}}{T_{p}} \gamma_{p s}+\frac{p_{s p}}{T_{p}} \gamma_{s p}\right)^{2}}\right) .
\end{aligned}
$$

We first investigate the scenario where both $p_{s p}$ and $p_{p}$ are positive. Setting (50) and (51) to zero, we obtain

$$
\frac{\gamma_{p p}}{T_{p}}+\frac{\frac{p_{s p}^{2} \gamma_{p s} \gamma_{s p}^{2}}{T_{p}^{3}}}{\left(\frac{p_{p}}{T_{p}} \gamma_{p s}+\frac{p_{s p}}{T_{p}} \gamma_{s p}\right)^{2}}=\frac{\frac{p_{p}^{2} \gamma_{p s}^{2} \gamma_{s p}}{T_{p}^{3}}}{\left(\frac{p_{p}}{T_{p}} \gamma_{p s}+\frac{p_{s p}}{T_{p}} \gamma_{s p}\right)^{2}}
$$

which results in

$$
\frac{p_{p}}{T_{p}}=\chi \frac{p_{s p}}{T_{p}}
$$

where

$$
\chi=\frac{\sqrt{\gamma_{p p} \gamma_{s p}-\gamma_{p p} \gamma_{p s}+\gamma_{p s} \gamma_{s p}}+\gamma_{p p}}{\gamma_{s p}-\gamma_{p p}} \cdot \frac{\gamma_{s p}}{\gamma_{p s}} .
$$

In order to guarantee $p_{s p}^{*}>0, \chi>0$ must be satisfied. Thus, we have $\gamma_{s p}>\gamma_{p p}$. Substituting (53) into (50), we can get

$$
\begin{aligned}
\frac{p_{s p}}{T_{p}}= & \frac{\chi \gamma_{p s}+\gamma_{s p}}{\chi^{2} \gamma_{p p} \gamma_{p s}+\chi \gamma_{p p} \gamma_{s p}+\chi \gamma_{p s} \gamma_{s p}} \\
& \cdot\left(\left(\frac{\beta \theta_{p}}{\ln 2 \omega_{1}} \cdot \frac{\chi^{2} \gamma_{p s}^{2} \gamma_{s p}}{\left(\chi \gamma_{p s}+\gamma_{s p}\right)^{2}}\right)^{\frac{\ln 2}{\ln 2+\theta_{p} T_{p}}}-1\right) .
\end{aligned}
$$

In the case where (55) is negative, $\frac{p_{s p}}{T_{p}}$ should be set to zero. When $\gamma_{s p} \leq \gamma_{p p}$, it can be also proved that $p_{s p}^{*}=0$.
For these two cases, the allocated power over the PT $\rightarrow$ ST adheres to the QoS-based water-filling policy shown as

$$
\frac{p_{p}}{T_{p}}=\left(\frac{\theta_{p} T_{p} \beta}{\ln 2 T_{p} \omega_{1}}\right)^{\frac{\ln 2}{\ln 2+\theta_{p} T_{p}}} \cdot\left(\frac{1}{\gamma_{p p}}\right)^{\frac{\theta_{p} T_{p}}{\ln 2+\theta_{p} T_{p}}}-\frac{1}{\gamma_{p p}} .
$$

Thus, the optimality of solution $p_{p}^{*}$ and $p_{s p}^{*}$ in (25) and (26) is proved.

\section{Endnote}

${ }^{1}$ The unit for service rate and the effective capacity is bits per frame.

\section{Acknowledgements}

This work was supported by the National Science Foundation of China with Nos. 61571272, 61201269, and 61403230. The authors would like to thank the anonymous reviewers for their constructive comments, which helped a lot to improve the presentation of this paper.

\section{Authors' contributions}

In this research paper, the authors proposed an resource allocation algorithm. All authors read and approved the final manuscript.

\section{Competing interests}

The authors declare that they have no competing interests.

\section{Publisher's Note}

Springer Nature remains neutral with regard to jurisdictional claims in published maps and institutional affiliations.

Received: 27 December 2016 Accepted: 21 May 2017

Published online: 15 June 2017

\section{References}

1. S Jayaweera, G Vazquez-Vilar, C Mosquera, Dynamic spectrum leasing: a new paradigm for spectrum sharing in cognitive radio networks. IEEE Trans. Veh. Technol. 59(5), 2328-2339 (2010)

2. O Simeone, I Stanojev, S Savazzi, Y Bar-Ness, U Spagnolini, R Pickholtz, Spectrum leasing to cooperating secondary ad hoc networks. IEEE J Sel. Areas Commun. 26(1), 203-213 (2008)

3. M Naeem, A Anpalagan, M Jaseemuddin, D Lee, Resource allocation techniques in cooperative cognitive radio networks. IEEE Commun. Surv. Tutor. 16(2), 729-744 (2014)

4. A Ahmad, S Ahmad, M Rehmani, N Hassan, A survey on radio resource allocation in cognitive radio sensor networks. IEEE Commun. Surv. Tutor. 17(2), 888-917 (2015)

5. A Gavili, S ShahbazPanahi, Optimal spectrum leasing and resource sharing in two-way relay networks. IEEE Trans. Sig. Process. 62(19), 5030-5045 (2014)

6. W Lu, Y Gong, S Ting, X Wu, N Zhang, Cooperative OFDM relaying for opportunistic spectrum sharing: protocol design and resource allocation. IEEE Trans. Wireless Commun. 11(6), 2126-2135 (2012)

7. M Hafeez, J Elmirghani, Analysis of dynamic spectrum leasing for coded bi-directional communication. IEEE J. Sel. Areas Commun. 30(8), 1500-1512 (2012)

8. M Pandian, M Sichitiu, H Dai, Optimal resource allocation in random access cooperative cognitive radio networks. IEEE Trans. Mobi. Compu. 14(6), 1245-1258 (2015)

9. A Ghosh, S Sarkar, Quality-sensitive price competition in secondary market spectrum oligopoly--single location game. IEEE/ACM Trans. Netw. 24(3), 1894-1907 (2016)

10. L Duan, L Gao, J Huang, Cooperative spectrum sharing: a contract-based approach. IEEE Trans. Mobi. Comput. 13(1), 174-187 (2014)

11. B Nazari, A Jamalipour, Contract-auction based distributed resource allocation for cooperative communications. IET Commun. 10(9), 1087-1095 (2016)

12. S Toroujeni, S Sadough, S Ghorashi, Spectrum leasing for OFDM-based cognitive radio networks. IEEE Trans. Vehi. Tech. 62(5), 2131-2139 (2013) 
13. A Alsharoa, H Ghazzai, E Yaacoub, M Alouini, A Kamal, Joint bandwidth and power allocation for MIMO two-way relays-assisted overlay cognitive radio systems. IEEE Trans. Cogni. Commun. and Net. 1(4), 383-393 (2015)

14. X Feng, G Sun, X Gan, F Yang, X Tian, X Wang, M Guizani, Cooperative spectrum sharing in cognitive radio networks: a distributed matching approach. IEEE Trans. Commun. 62(8), 2651-2664 (2014)

15. M Shamaiah, S Lee, S Vishwanath, H Vikalo, Distributed algorithms for spectrum access in cognitive radio relay networks. IEEE J Sel. Areas Commun. 30(10), 1947-1957 (2012)

16. I Balapuwaduge, F Li, A Rajanna, M Kaveh, Channel occupancy-based dynamic spectrum leasing in multichannel CRNs: strategies and performance evaluation. IEEE Trans. Commun. 64(3), 1313-1328 (2016)

17. L Wang, V Fodor, Dynamic cooperative secondary access in hierarchical spectrum sharing networks. IEEE Trans. Wireless Commun. 13(11), 6068-6080 (2014)

18. W Li, X Cheng, T Jing, $X$ Xing, in Proc. IEEE INFOCOM. Cooperative multi-hop relaying via network formation games in cognitive radio networks, (Turin, 2013)

19. D Wu, R Negi, Effective capacity: a wireless link model for support of quality of service. IEEE Trans. Wireless Commun. 2(4), 630-643 (2003)

20. J Tang, X Zhang, Quality-of-service driven power and rate adaptation over wireless links. IEEE Trans. Wireless Commun. 6(8), 3058-3068 (2007)

21. Y Li, L Liu, H Li, J Zhang, Y Li, Resource allocation for delay-sensitive traffic over LTE-advanced relay networks. IEEE Trans. Wirel. Commun. 14(8), 4291-4303 (2015)

22. K Khan, T Le-Ngoc, L Le, Optimal resource allocation for buffer-aided relaying with statistical QoS constraint. IEEE Trans. Commun. 64(3), 959-972 (2016)

23. R Rockafellar, R Wets, Stochastic convex programming: basic duality. Pac. J. Math. 62(1), 173-195 (1976)

24. P Kall, S Wallace, Stochastic programming. (Wiley, New Jersey, 1994)

25. S Yang, Z Sheng, J McCann, K Leung, Distributed stochastic cross-layer optimization for multi-hop wireless networks with cooperative communications. IEEE Trans. Mobile Compu. 13(10), 2269-2282 (2014)

26. D Wang, X Wang, X Cai, Optimal power control for multi-user relay networks over fading channels. IEEE Trans. Wireless Commun. 10(1), 199-207 (2011)

27. X Wang, G Giannakis, Power-efficient resource allocation for time-division multiple access over fading channels. IEEE Trans. Inf. Theory. 54(3), $1225-1240(2008)$

28. J Laneman, D Tse, G Wornell, Cooperative diversity in wireless networks: efficient protocols and outage behavior. IEEE Trans. Inf. Theory. 50(12), 3062-3080 (2004)

29. J Laneman, G Wornell, Distributed space-time-coded protocols for exploiting cooperative diversity in wireless networks. IEEE Trans. Inf. Theory. 49(10), 2415-2425 (2003)

30. C-S Chang, Performance guarantees in communication networks, (Springer-Verlag London, 2000)

31. L Zadeh, Optimality and non-scalar-valued performance criteria. IEEE Trans. Autom. Control. 8(1), 59-60 (1963)

32. K Miettinen, Nonlinear multiobjective optimization. (Kluwer Academic Publishers, Dordrecht, 1999)

33. S Boyd, L Vandenberghe, Convex optimization. (Cambridge University Press, 2004)

34. S Boyd, A Mutapcic, Subgradient methods, notes for EE364, Standford University, Winter 2006-07

35. Y Ma, H Zhang, D Yuan, D Jiang, Power-efficient resource allocation with QoS guarantees for TDMA fading channels. Wirel. Commun. and Mobi. Comput. 12(11), 1023-1036 (2012)

36. A Goldsmith, Wireless communications. (Cambridge University Press, Cambridge, 2005)

37. V Solo, X Kong, Adaptive signal processing algorithms: stability and performance. (Prentice Hall, New Jersey, 1995)

\section{Submit your manuscript to a SpringerOpen ${ }^{\circ}$ journal and benefit from:}

\section{- Convenient online submission}

Rigorous peer review

- Open access: articles freely available online

- High visibility within the field

- Retaining the copyright to your article

Submit your next manuscript at $\gg$ springeropen.com 